\title{
Behavioural and emotional well-being of children following non-directive play with school staff
}

\author{
Donna L. Ewing ${ }^{\mathrm{a}}$, Jeremy J. Monsen ${ }^{\mathrm{b}}$, and Maria Kwoka ${ }^{\mathrm{c}}$ \\ ${ }^{a}$ University of Sussex, School of Psychology, Pevensey Building, Falmer, Brighton, England, \\ $B N 19 Q H$ \\ ${ }^{b}$ East London Consortium of Educational Psychologists, Education Support Services, \\ Children and Young People Service, Waltham Forest Council Summerfield Centre, 99 Leyton \\ Green Road, London, England, E10 6DB \\ ${ }^{\mathrm{c}}$ Kent Educational Psychology Services, Joynes House, New Road, Gravesend, Kent, \\ England, DA11 OAT
}

Emails: ewing.donna@gmail.com*; Jeremy.Monsen@virgin.net, Maria.Kwoka@kent.gov.uk

March 2013

\footnotetext{
* All correspondence concerning this article should be addressed to Donna Ewing, University of Sussex, School of Psychology, Pevensey Building, Falmer, Brighton, England, BN1 9QH Email: ewing.donna@gmail.com; Tel: 07716307610
} 
This action research project considered whether significant improvements in child and young person behavioural and emotional mental health could be achieved using school-based play workers as opposed to qualified therapists. This was seen as being an important practice question as access to qualified play therapists was severely restricted with long waiting lists. The Strengths and Difficulties Questionnaire (SDQ; Goodman, 1997 \& 1999) was used as a pre- and post-intervention measure to identify any changes following non-directive play sessions with school staff. Significant improvements were found across all SDQ scales, with the most marked improvement observable in children and young people identified as having a high need for intervention. Number of play sessions attended and age group did not significantly affect $S D Q$ scores according to teacher and child/young person ratings. Parent $S D Q$ ratings indicated greater success of the play intervention for children aged between 3 and 8 years compared with children aged between 11 and 15 years.

Key words: non-directive play; therapy; school-based; mental health; Strengths and Difficulties Questionnaire. 
There are increasing numbers of children and young people presenting with mental health issues in the United Kingdom, resulting in growing waiting lists for Child and Adolescent Mental Health Services (CAMHS, British Medical Association, 2006). Kerfoot, Panayiotopoulos, and Harrington (2004) found that 49\% of Local Authorities had CAMHS waiting lists of one to five months, and a further $18 \%$ had a six month waiting list. Without early interventions, the mental health of children and young people may get worse (Ginsburg, 2009). For this reason it is important to investigate how best to approach the waiting list issue and to help minimise the risk of children and young people's mental health difficulties escalating and becoming more entrenched.

To address these issues, alternative options were considered for meeting the needs of children and young people with behavioural and emotional difficulties, such as those presenting with anxiety, stress/depression, behavioural, conduct, cognitive and social

difficulties. A pilot study indicated success in using non-directive play as an intervention, so the current study was set up to take this forward by training teachers, Special Educational Needs Coordinators (SENCOs), and teaching assistants in non-directive play skills, supervised by fully qualified play therapists. For the remainder of this paper, young people will be included within the terms 'child' or 'children'.

Non-directive play therapy (also referred to as child-centered play therapy) is an approach used with children who have a range of social, emotional and behavioral needs. It is based on the assumption that play is in effect the child's "core language", using toys and words to express their perceptions of their experiences and how they currently perceive themselves, others and the world. The toys chosen by the child "speak" to the therapist and communicate the child's current set of perceptions. Within a play session, and over the course of working with the child, themes and patterns emerge. This gives the therapist 
insight into the child's experiences, thoughts, feelings and interpretations of their world (Ray, Bratton, Rhine \& Jones, 2001).

In a non-directive manner the therapist aims to help the child externalise their perceptions of their experiences, themselves, others and the world so they can be 'worked on'. The assumption is that helping children externalize such perceptions via the use of toys and play assists them to cognitively re-structure unhelpful thoughts, feelings and beliefs leading to changes in thinking and behavior at home and at school. A core goal for the play therapist, once robust themes and patterns have emerged, is to help the child to review and work through their experiences. Often this occurs through the use of metaphor via the therapist "talking" through the toys. In this way the therapist can engage in a dialogue which helps the child to understand and rework the problem or issue (Ray et al., 2001).

The effect of training and supervising practitioners to implement play sessions could provide a robust intervention for those unable to access CAMHS immediately. The current study sought to investigate whether similar benefits to that of play therapy interventions were found for children who engage in non-directive play with trained and supervised school-staff. Research indicates positive effects of play therapy interventions on children's behaviour with reference to a range of problem areas such as anxiety and behavioural problems (Fall, Navelski, \& Welch, 2002; Draper, White, O’Shaughnessy, Flynt \& Jones, 2001; Danger \& Landreth, 2005; Packman \& Bratton, 2003). For example, play therapy has been successful in reducing anxiety symptoms in children with special educational needs (Fall et al., 2002) and in reducing both anxiety and depression in discouraged or struggling children (Draper et al., 2001) and preadolescent children (Packman \& Bratton, 2003). In addition, a play intervention for children with behavioural/conduct issues was successful in reducing teachers' stress about the children's anxiety, withdrawal behaviours and emotional distress (Ray, Schottelkorb \& Tsai, 2007). Play was ineffective in reducing anxiety in children with 
Speech and Language Difficulties (Danger \& Landreth, 2005) although Danger and Landreth (2005) acknowledge that the anxiety of this group was low pre- intervention, which could explain why there was not a significant reduction. This highlights the importance of investigating the level of intervention need prior to the intervention, which the current study aimed to achieve by identifying whether children had "low", "medium" or "high" intervention needs and considering the effectiveness of the intervention for each of these groups.

Play therapy has been successful in improving children's behavioural problems such as hyperactivity, inattention, cognitive problems, aggression, and conduct problems (Draper et al., 2001; Packman \& Bratton, 2003; Post, McAllister, Sheely, Hess \& Flowers, 2004; Ray, Schottelkorb \& Tsai, 2007), whilst improving the child's presentation of anxiety and depression, and improving their adaptive skills, including social skills, adaptability, leadership and study skills (Post et al., 2004). In a school-based play therapy intervention, teachers reported significant reductions in aggressive problem behaviours for those children who received the intervention compared to those in the control group (Ray, Blanco, Sullivan \& Holliman, 2009). Packman and Bratton (2003) found moderate (but non-significant) effect sizes for a reduction in aggression and delinquent behaviour in preadolescents following play therapy. Although these various research studies were limited by small sample sizes, the findings are suggestive that play interventions can be successful in reducing both anxiety and behavioural difficulties. The current study investigated whether a non-directive play intervention similarly improves children's emotional responses and behavioural difficulties, using a larger, more representative sample.

There is mixed research evidence regarding the importance of the number of play therapy sessions required for the intervention to be successful. Muro, Ray, Schottelkorb, Smith and Blanco (2006) considered the effects of long-term play therapy for children with 
behavioural or emotional problems, measuring behavioural problems at pre- (0 sessions), mid- (16 sessions) and post- play therapy (32 sessions). They found a significant reduction $\underline{\text { in }}$ behavioural problems from pre- to post- play therapy. Short-term interventions may be similarly effective as long-term interventions. For instance, Fall et al. (2002) found significant improvements in child anxiety following only six weekly play therapy sessions. The current study will investigate whether the number of play sessions has an impact on the success of the intervention to clarify the apparent discrepancy between these two sets of findings.

\section{Purpose of the Current Study}

The purpose of the current study was to investigate whether non-directive play leads to a significant improvement in child SDQ scores when school staff are trained and supervised by fully qualified play therapists to undertake play interventions. The results of this study could lead to the wider implementation of play interventions led by school staff and reduce the demand on CAMHS if the staff-led intervention proves to be successful. This could reduce waiting lists and enable CAMHS staff to focus on the most severe clients. Further, this research contributes to the growing knowledge base regarding play interventions since there is currently little information available regarding general play interventions as opposed to play therapy or therapeutic play undertaken by qualified play therapists directly.

To investigate the success of this school-based staff led intervention, changes in prosocial behaviour, hyperactivity/inattention, emotional symptoms, peer problems and conduct problems were measured pre- and post the non-directive play intervention using the SDQ. The research question addressed in this paper is whether non-directive play with $\underline{\text { school-based staff leads to a statistically significant improvement in child prosocial }}$ behaviour, and a statistically significant reduction in hyperactivity/inattention, emotional 
symptoms, peer problems and conduct problems according to teacher, parent/carer and child SDQ ratings.

\section{METHOD}

\section{Participants}

Children (aged 3 years 10 months to 14 years 8 months) identified with emotional and/or behavioural difficulties $(\mathrm{N}=254)$, according to teachers ratings on the CAMHS

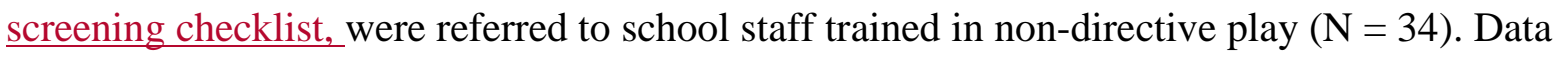
was discarded for one child who only participated in 2 sessions, leaving a sample of 253 children $($ Female $=81 ;$ Male $=172)$. Note, this figure represents the total number of children included in the study, but data was not available from every parent/carer, teacher and child for each child included in the study. The mean age of participants was 8 years 6 months (mean $=101.59$ months, $\mathrm{SD}=29.14)$ and the mean number of sessions attended was 13.78 $(\mathrm{SD}=8.34)$, with a range of 3 to 62 sessions. For the purposes of data analysis, SDQ data was available for 245 children according to teacher ratings, 189 children according to parent/carer ratings, and 138 children according to child self-ratings. The play workers (33 female; 1 male) included seven teachers, two nursery nurses, four higher level teaching assistants, eleven other teaching assistants, three family liaison officers, and seven learning mentors.

\section{Instruments}

Strengths and Difficulties Questionnaire (SDQ) (Goodman, 1997 \& 1999). This questionnaire comprises 25 questions, measuring scales of hyperactivity, emotional symptoms, conduct problems, peer problems and prosocial behaviour. Responses of "not true", "somewhat true" and "certainly true" were scored as 0,1 or 2 respectively, or were 
reverse scored where applicable. Prosocial behaviour had a maximum score of 10 and the sum of scores for the difficulties scales gave a maximum score of 40 for total difficulties.

The SDQ is widely used to assess the mental health of children, and is recommended for using for child assessments by the CAMHS Outcomes Research Consortium (CORC, n.d.). The SDQ has good predictive validity for a number of child difficulties including depression and anxiety, is the most efficient assessment of attention disorders, and is a strong predictor of disruptive behaviours (Warnick, Weersing, Scahill \& Woolston, 2009). It is useful in assessing mental health issues in children, particularly since it is short, comprehensible and reliable (Vogels, Crone, Hoekstra, \& Reijneveld, 2009; Janssens \& Deboutte, 2009).

Mellor and Stokes (2007) found that children, teachers and parents/carers respond significantly differently from one another on the SDQ, suggesting that data should be collected from all three sources for optimal predictive validity. The SDQ has sound test-retest reliability (parent $r=.52$ to .85 ; teacher $r=.64$ to .82 ; child $r=.66$ to .82 ) at $p<0.01$ (Mellor, 2004).

Demographic data - child. Age, gender, number of sessions, and therapeutic play practitioner worked with.

Demographic data - play worker. Gender and school role.

\section{Procedure}

Ethical approval was gained for this study through the Local Authority Ethics Committee for Applied Research. School-based staff were trained in nondirective play by play therapists, which included 15 days of training. The training involved studying 3-4 modules across a number of months and the school-based staff were awarded a certificate 
following successful completion of the training. Each of these staff practised non-directive play alongside another role in the school.

Children exhibiting difficult behaviours or emotional problems, as determined by their teacher-rated scores on the CAMHS screening checklist, were referred for the play intervention by their school SENCO. Prior to the onset of the play sessions, the play worker met with the child and his or her parents/carers to discuss the intervention and to complete the pre-intervention SDQ. The child's teacher completed the pre-intervention teacher SDQ. Oneto-one nondirective play sessions took place in a designated room in the child's school.

During the non-directive play, the therapist helped the child to externalise their perceptions of their experiences, themselves, others and the world using toys and play, helping the child to cognitively restructure any unhelpful thoughts, feelings and beliefs. At the end of the intervention, the SDQ was completed by the child, parent/carer and teacher, and the play workers sent the data to the local authority for analysis.

\section{Data Analysis}

Paired samples t-tests and effect sizes were conducted to assess the significance of any change in scores on the SDQ from pre- to post- play intervention according to child, parent/carer and teacher SDQ scores. A one-way ANOVA tested whether there were significant differences in the scores according to child, parent/carer and teacher ratings of the SDQ for each of the scales. The data was then split according to total difficulties score, and ttests and effect sizes were generated for children with "low", "medium" and "high" difficulties. A total difficulties score between zero and fifteen indicated a low need for intervention since the child demonstrated minimal hyperactivity and few emotional, conduct or peer problems. A total difficulties score between sixteen and nineteen indicated a moderate need for intervention since the child demonstrated some hyperactivity, emotional, conduct or 
peer problems. A total difficulties score greater than twenty indicated a high need for intervention since the child demonstrated high levels of difficulties.

To minimise the risk of conducting the Type I error, Tukey's Honesty Significance Test (HSD) was used for ANOVA statistics, and Bonferroni corrections were used for the ttest statistics. After applying the Bonferroni correction, an alpha level of $p<.008$ was adopted for the t-tests, based on six t-tests conducted for the pre- and post-intervention results according to each scorer within the different levels of intervention need. As recommended by Dunlap, Cortina, Vaslow and Burke (1996) effect sizes were calculated using Cohen's d according to the original mean and standard deviation scores to avoid generating an overestimated effect size. This paper adopted Cohen's (1988) criteria of 0.2 indicating a small effect size, 0.5 a medium effect size, and 0.8 a large effect size.

\section{RESULTS}

\section{Teacher, Parent/Carer and Child SDQ Ratings}

SDQ data completed by all three scorers (teacher, parent/carer, and child) were gathered for 109 children pre- and post- play intervention (Female: $\mathrm{N}=37$; Male: $\mathrm{N}=72$ ) with an average age of 9 years $(M=107.61$ months; $S D=28.94)$, and an average attendance at 14 play sessions $(\mathrm{M}=13.82 ; \mathrm{SD}=8.46)$. A one-way ANOVA was conducted for each of the SDQ scales to investigate any differences in teacher, parent/carer and child scoring.

Results revealed a significant difference between scorer on each scale, except for emotional symptoms and conduct problems pre- non-directive play. For prosocial behaviour, there was a significant difference between scorer both pre- $(\mathrm{F}(2,324)=48.93, \mathrm{p}>.001$, $)$ and post- non-directive play $(\mathrm{F}(2,324)=34.66, \mathrm{p}<.001)$. The Tukey HSD post-hoc test revealed that teachers rated child prosocial behaviour significantly lower than parents/carers and 
children both pre- (parent: $\mathrm{p}<.001, \mathrm{~d}=0.82$; child: $\mathrm{p}<.001, \mathrm{~d}=1.31$ ) and post- nondirective play (parent: $\mathrm{p}<.001, \mathrm{~d}=0.72$; child: $\mathrm{p}<.001, \mathrm{~d}=1.12$ ). Parents/carers also rated the child's prosocial behaviour significantly lower than children pre- non-directive play $(\mathrm{p}<$ $.01, \mathrm{~d}=0.47)$.

For hyperactivity/inattention, there was a significant difference between scorer both pre- $(\mathrm{F}(2,322)=3.80, \mathrm{p}<.05)$ and post- non-directive play $(\mathrm{F}(2,322)=3.92, \mathrm{p}<.05)$. The Tukey HSD post-hoc test revealed that parents rated hyperactivity/inattention significantly higher than teachers at pre-intervention $(\mathrm{p}<.05, \mathrm{~d}=0.33)$ and significantly higher than children at post- intervention $(\mathrm{p}<.05, \mathrm{~d}=0.27)$

There was a significant difference between scorer on emotional symptoms score postintervention $(\mathrm{F}(2,322)=3.72, \mathrm{p}<.05)$ which the Tukey HSD revealed that teachers rated emotional symptoms significantly lower than children $(\mathrm{p}<.05, \mathrm{~d}=0.37)$.

There was a significant difference between scorers on conduct problems at postintervention, $\mathrm{F}(2,322)=3.53, \mathrm{p}<.05$. The Tukey HSD revealed that parents rated conduct problems significantly higher than teachers at post-intervention $(\mathrm{p}<.05, \mathrm{~d}=0.34)$.

For peer problems, there was a significant difference between scorer both pre$(\mathrm{F}(2,322)=5.19, \mathrm{p}<.01)$ and post- non-directive play $(\mathrm{F}(2,322)=5.31, \mathrm{p}<.01)$. The Tukey HSD revealed that children rated peer problems significantly less than parents/carers both pre- intervention $(\mathrm{p}<.01, \mathrm{~d}=0.46)$ and post- intervention $(\mathrm{p}<.01, \mathrm{~d}=0.44)$.

The total difficulties score differed significantly between scorer pre- $(\mathrm{F}(2,322)=$ $4.66, \mathrm{p}<.01)$ and post- non-directive play $(\mathrm{F}(2,322)=4.88, \mathrm{p}<.01)$, which the Tukey HSD revealed that teachers rated the total difficulties significantly less than parents/carers pre- $(\mathrm{p}<$ $.05, \mathrm{~d}=0.39)$ and post- intervention $(\mathrm{p}<.01, \mathrm{~d}=0.39)$.

\section{SDQ Bandings}


For the remainder of the analysis, children were identified as having "low", "medium" or "high" intervention needs according to their pre-intervention total difficulty SDQ scores. The following results sections refer to the teacher, parent/carer and child SDQ ratings for children with a high intervention need. Results for children with medium and low intervention needs are summarised in Table 1.

\section{Total Difficulties Score 20 - 40 (high intervention need): Teacher rating}

A total difficulties score greater than twenty indicated a high need for intervention since the child demonstrated high levels of difficulties. Of the 245 teachers SDQ scores, total difficulties score was greater than twenty for 91 children (Female: 21; Male: 70) with a mean score of $23.41(\mathrm{SD}=3.20)$, an average age of 8 years 5 months $(\mathrm{M}=101.37, \mathrm{SD}=31.43)$ and an average attendance of 16 play sessions $(M=15.64, S D=9.83)$.

The paired sampled t-test revealed a statistically significant increase in prosocial behaviour, $\mathrm{t}(90)=5.80, \mathrm{p}<.001, \mathrm{~d}=0.63$, and a statistically significant reduction in hyperactivity, $\mathrm{t}(90)=-5.43, \mathrm{p}<.001, \mathrm{~d}=0.62$; emotional symptoms, $\mathrm{t}(90)=-5.75, \mathrm{p}<.001$, $\mathrm{d}=0.68$; conduct problems, $\mathrm{t}(90)=-5.48, \mathrm{p}<.001, \mathrm{~d}=0.63$; and peer problems, $\mathrm{t}(90)=-$ $6.59, \mathrm{p}<.001, \mathrm{~d}=.66$, and a significant reduction in total difficulties, $\mathrm{t}(90)=-8.92, \mathrm{p}<.001$, $\mathrm{d}=1.19$.

\section{Total Difficulties Score 20 - 40 (high intervention need): Parent/Carer rating}

Of the 189 parents/carers who completed the SDQ, total difficulties score was greater than twenty for 90 children (Female: 28; Male: 62), with a mean score for children of 24.99 $(\mathrm{SD}=3.46)$, an average age of 8 years 10 months $(\mathrm{M}=106.19, \mathrm{SD}=28.79$, and an average attendance of 15 play sessions $(\mathrm{M}=14.56, \mathrm{SD}=9.83)$. 
The paired samples t-test revealed a statistically significant increase in prosocial behaviour, $\mathrm{t}(89)=4.31, \mathrm{p}<.001, \mathrm{~d}=0.37$, and a statistically significant reduction in hyperactivity, $\mathrm{t}(89)=-6.10, \mathrm{p}<.001, \mathrm{~d}=0.63$, emotional symptoms, $\mathrm{t}(89)=-7.98, \mathrm{p}<.001$, $\mathrm{d}=0.79$, conduct problems, $\mathrm{t}(89)=-5.23, \mathrm{p}<.001, \mathrm{~d}=0.46$, peer problems, $\mathrm{t}(89)=-2.78, \mathrm{p}$ $<.008, \mathrm{~d}=0.29$, and total difficulties, $\mathrm{t}(89)=-7.92, \mathrm{p}<.001, \mathrm{~d}=0.96$,

\section{Total Difficulties Score 20 - 40 (high intervention need): Child rating}

Of the 138 children who completed the SDQ, total difficulties score was greater than twenty for 61 children (Female: 24; Male: 37 ), with a mean score of $23.56(\mathrm{SD}=3.38$ ), an average age of 9 years 9 months $(M=116.67, S D=29.18)$, and an average attendance of 15 play sessions $(\mathrm{M}=14.83, \mathrm{SD}=7.98)$.

The paired samples t-test revealed a statistically significant increase in prosocial behaviour, $\mathrm{t}(60)=2.78, \mathrm{p}<.008, \mathrm{~d}=0.34$, and a statistically significant reduction in hyperactivity, $\mathrm{t}(60)=-4.27, \mathrm{p}<.001, \mathrm{~d}=0.59$; emotional symptoms, $\mathrm{t}(60)=-4.75, \mathrm{p}<.001$, $\mathrm{d}=0.59$; conduct problems, $\mathrm{t}(60)=-6.17, \mathrm{p}<.001, \mathrm{~d}=0.67$; and peer problems, $\mathrm{t}(60)=-$ $4.09, \mathrm{p}<.001, \mathrm{~d}=0.62$, and a reduction in total difficulties, $\mathrm{t}(60)=-8.05, \mathrm{p}<.001, \mathrm{~d}=1.20$.

[INSERT TABLE 1]

\section{Impact of the intervention}

Impact scores for prosocial behaviour and total difficulties were calculated by subtracting the pre-intervention SDQ score from the post-intervention SDQ score. Figure 1 indicates an improvement in prosocial behaviour and a reduction in difficulties following the play intervention. 
[INSERT FIGURE 1]

\section{Age Group Performance}

Participants were divided into three age groups ( 3 years to 7 years 11 months; 8 years to 10 years 11 months; and 11 years to 14 years 11 months). A one-way ANOVA was conducted to assess any differences in the impact of the play intervention according to the child's age and revealed no significant differences between age group for teacher, parent/carer or child $(\mathrm{p}>.05)$ impact scores for total difficulties, nor for teacher or child impact scores for prosocial behaviour $(\mathrm{p}>.05)$. A significant difference was found between age groups for parent/carer impact scores on prosocial behaviour, $\mathrm{F}(2,185)=4.41, \mathrm{p}<.05$. The Tukey HSD post-hoc test revealed that the intervention had a significantly greater impact in improving prosocial behaviour for those in the youngest age group than those in the oldest age group according to parent/carer ratings, $\mathrm{p}<.01, \mathrm{~d}=0.56$.

\section{Number of Sessions}

Participants were divided into three groups according to the number of sessions they attended: "less than nine", "ten to thirteen", or "over fourteen" sessions. A one-way ANOVA was conducted to assess whether the number of play sessions attended by the child had an effect on the impact score. Results indicated no significant differences for teacher, parent/carer or child impact scores according to number of sessions attended $(\mathrm{p}>0.025)$.

\section{DISCUSSION}

The results of this study suggest that play sessions with school-based staff working under the supervision of play therapists may be successful in improving child prosocial behaviour and reducing difficulties in hyperactivity/inattention, emotional symptoms, 
conduct problems, peer problems and total difficulties according to teacher, parent/carer and child SDQ ratings. This has implications for the way that children with behavioural, emotional or social difficulties are worked with. Implementing play interventions through school-based staff could greatly reduce the time that the child waits for an intervention, and in turn minimises the risk of their difficulties intensifying (Ginsburg, 2009). In addition, using this school-staff led intervention for less severe children could enable CAMHS to focus on the most severe clients.

However, the current study was limited in that it was not possible to adopt a classical experimental design, assigning non-intervention children to a control group. This was because the study aimed to help children unable to access therapy through CAMHS. Therefore, to use another wait-list as a control would have been counter to the aims of the study. Further research is required to compare the results of children engaged in these play $\underline{\text { sessions to children in a wait-list control group to determine whether it is the intervention }}$ itself that is responsible for the improvement in children's difficulties found in the current study. Since this was not possible in the current study, separate analyses were conducted for children identified as having "high", "medium" or "low" intervention needs. The larger and more significant effects seen in children with a high intervention need, compared with low and medium intervention needs, may suggest that the intervention itself led to significant improvements in prosocial behaviour and reductions in difficulties, although further research with a control group is required to confirm this tentative conclusion.

There were significant differences between teacher, parent/carer and child ratings on each of the subscales pre- and post- intervention, except for emotional symptoms and conduct problems pre-intervention. Teachers scored children significantly lower than parents/carers did on prosocial behaviour, hyperactivity/inattention, and total difficulties, and scored 
children's emotional symptoms significantly lower than the child's self-rating. This finding supports Ray et al.'s (2009) conclusion- that teachers rated children more positively than parents did following the intervention, with the exception of ratings of prosocial behaviour in the current study. Future research could consider whether children find it difficult to generalise from the play intervention in school to settings outside of the school, such as at home. Such research might look to clarify why parents/carers see less of an improvement in their children than the teachers. In the current study, parents/carers scored their child significantly lower than the child's self-rating on prosocial behaviour, and the child rated their hyperactivity/inattention and peer problems significantly lower than their parent/carer.

The inconsistencies across scorer ratings highlight the importance of gathering data from a range of sources to ensure results accurately reflect the child's strengths and difficulties, whilst highlighting the danger of self-report data, the importance of using a variety of sources and the need for sufficient sample sizes (Mellor \& Stokes, 2007). Regardless of the inconsistencies across scorers' ratings, the results suggest there is an overall improvement in prosocial behaviour and a reduction in child difficulties following the play intervention.

It is important to consider the effect of any conflicting roles of school staff where they deal with class discipline as well as the play intervention. Since the play sessions seemed to be successful in reducing child difficulties and improving their prosocial behaviour in this study, it is argued that any effect of conflicting staff roles is minimal. However, this is a potential limitation to the current study and it may be beneficial to conduct further research comparing the results of this study to that of another using the same play intervention but with play workers previously unknown to the children.

Results from this study suggest that the play interventions were more successful for children identified as "high intervention need" in terms of improving prosocial behaviour and 
reducing hyperactivity/inattention, emotional symptoms, conduct problems, peer problems and total difficulties, irrespective of scorer. For "low intervention need" children, the play intervention appears to be successful in improving prosocial behaviour according to teacher and parent/carer ratings, although did not seem to be successful in reducing hyperactivity/inattention, emotional symptoms, conduct problems or peer problems. It is likely that the "low intervention need" group had limited potential for improvement given that they were already functioning within the "normal" range prior to the intervention.

This study suggests that interventions were more successful for children between the ages of 3 and 8 compared with children between the ages of 11 and 15, according to parent/carer ratings. Unlike previous research findings (Muro et al, 2006), the current study found that the success of the play intervention did not change significantly according to the number of sessions attended, although interventions in the current study were relatively short $($ mean $=13.78$ sessions $)$ and significant effects may be evident if children attended a greater number of play sessions.

In conclusion and according to the findings of the current study, play interventions using school-based practitioners can tentatively be concluded to be successful in significantly improving children's behaviours, with particular benefits noticeable for children with higher intervention needs. This $\underline{\text { suggests }}$ that school based play workers may make a significant difference to children with relatively high intervention needs. Training school-based staff in play techniques may therefore be a useful intervention in reducing difficulties, particularly in consideration of the long waiting list for CAMHS services, although further research adopting a control group is required to confirm this finding. Future research could compare the effectiveness of play using school-based practitioners against other interventions currently used within schools, as well as considering the long-term benefits of children engaging in play interventions with school-based staff. 


\section{REFERENCES}

BMA (2006). Child and Adolescent Mental Health: A guide for healthcare professionals.

London: British Medical Association: Board of Science.

CAMHS Outcomes Research Consortium (CORC). (n.d.). Getting Started-CORC+model.

Retrieved from www.corc.uk.net/joining/getting-started/corcplus/.

Cohen, J. (1988). Statistical power analysis for the behavioural sciences (2nd Ed.). Hillsdale, NJ: Lawrence Erlbaum Associates.

Danger, S., \& Landreth, G. (2005). Child-Centered Group Play Therapy with Children with Speech Difficulties. International Journal of Play Therapy, 14(1), 81-102.

Draper, K.; White, J.; O’Shaughnessy, T.E.; Flynt, M.; \& Jones, N. (2001). Kinder Training: Play-Based Consultation to Improve the School Adjustment of Discouraged Kindergarten and First Grade Students. International Journal of Play Therapy, 10(1), $1-30$.

Dunlap, W. P., Cortina, J. M., Vaslow, J. B., \& Burke, M. J. (1996). Meta-analysis of experiments with matched groups or repeated measures designs. Psychological Methods, 1(2), 170-177.

Fall, M; Navelski, L.F; \& Welch, K.K. (2002). Outcomes of a Play Intervention for Children Identified for Special Education Services. International Journal of Play Therapy, 11(2), 91-106.

Ginsburg, G.S. (2009). The Child Anxiety Prevention Study: Intervention Model and Primary Outcomes. Journal of Consulting and Clinical Psychology, 77(3), 580-587.

Goodman, R. (1997). The Strengths and Difficulties Questionnaire: A research note. Journal of Child Psychology and Psychiatry, 38(5), 581-586. 
Goodman, R. (1999). The extended version of the Strengths and Difficulties Questionnaire as a guide to child psychiatric caseness and consequent burden. Journal of Child Psychology and Psychiatry, 40(5), 791-799.

Janssens, A., \& Deboutte, D. (2009). Screening for psychopathology in child welfare: the Strengths and Difficulties Questionnaire (SDQ) compared with the Achenbach System of Empirically Based Assessment (ASEBA). European Child and Adolescent Psychiatry, 18(11), 691-700.

Kerfoot, M., Panayiotopoulos, C., \& Harrington, R. (2004). Social Services and CAMHS: A National Survey. Child and Adolescent Mental Health, 9(4), 162-167.

Koskelainen, M., Sourander, A., \& Kaljonen, A. (2000). The Strengths and Difficulties Questionnaire among Finnish school-age children and adolescents. European Child and Adolescent Psychiatry, 9(4), 277-284.

Mellor, D. (2004). Furthering the Use of the Strengths and Difficulties Questionnaire: Reliability with Younger Child Respondents. Psychological Assessment, 16(4), 396401.

Mellor, D.; \& Stokes, M (2007). The Factor Structure of the Strengths and Difficulties Questionnaire. European Journal of Psychological Assessment, 23(2), 105-112.

Muro, J.; Ray, D.; Schottelkorb, A.; Smith, M.R.; \& Blanco, P.J. (2006). Quantitative Analysis of Long-Term Child-Centered Play Therapy. International Journal of Play Therapy, 15(2), 35-58.

O’Connor, C., \& Stagnitti, K. (2011). Play, behaviour, language and social skills: The comparison of a play and non-play intervention within a specialist school setting. Research in Developmental Disabilities, 32, 1205-1211. 
Packman, J., \& Bratton, S.C. (2003). A School-Based Group Play/Activity Therapy Intervention with Learning Disabled Preadolescents Exhibiting Behaviour Problems. International Journal of Play Therapy, 12(2), 7-29.

Post, P.; McAllister, M.; Sheely, A.; Hess, B.; \& Flowers, C. (2004). Child-Centered Kinder Training for Teachers of Pre-School Children Deemed At-Risk. International Journal of Play Therapy, 13(2), 53-74.

Ray, D.C.; Blanco, P.J.; Sullivan, J.M.; \& Holliman, R. (2009). An Exploratory Study of Child-Centered Play Therapy with Aggressive Children. International Journal of Play Therapy, 18(3), 162-175.

Ray, D.C.; Schottelkorb, A.; \& Tsai, M.H. (2007). Play Therapy with Children Exhibiting Symptoms of Attention Deficit Hyperactivity Disorder. International Journal of Play Therapy, 16(2), 95-111.

Ray, D., Bratton, S., Rhine, T., \& Jones, L. (2001). The effectiveness of play therapy: Responding to the critics. International Journal of Play Therapy, 10(1), p. 85-108.

Vogels, A.G.C., Crone, M.R., Hoekstra, F., \& Reijneveld, I. (2009). Comparing three short questionnaires to detect psychosocial dysfunction among primary school children: a randomised method. BMC Public Health, 9, article 489.

Warnick, E.M., Weersing, V.R., Scahill, L. \& Woolston, J.L. (2009). Selecting measures for use in child mental health services: A scorecard approach. Administration and Policy in Mental Health and Mental Health Services Research, 36(2), 112-122. 